\section{Early neglect associated to prefrontal structural disconnectivity in schizophrenia}

Keywords:

Schizophrenia

Fractional anisotropy

Prefrontal

Maltreatment

Neglect

\section{Dear Editors:}

The contribution of maltreatment to the risk for schizophrenia may relate to alterations in structural brain connectivity reported in this syndrome, since early stress affects glia development (Delpech et al. 2016). Indeed, an inverse association between the severity of early adverse experiences and fractional anisotropy (FA) was described in schizophrenia (Poletti et al. 2015). However, the particular regions among which that connectivity is altered have not been identified. Since prefrontal cortex (PFC) may be specially affected by early social adversity (Davidson and McEwen 2012), its connectivity can be hampered by childhood maltreatment.

To this end, we collected early maltreatment data in 26 schizophrenia patients ( 12 first-episodes (FE) and 14 healthy controls. Of them, dMRI data were available in 19 patients (12 FE) and 13 controls, all included in a previous report on PFC connectivity (Molina et al. 2017). We assessed cognition with the Brief Assessment in Cognition in Schizophrenia Scale and Wisconsin Card test. Full data are shown in Supplementary material. Childhood experiences were assessed the Childhood Trauma Questionnaire (CTQ) (Bernstein et al. 2003), a Likert-type selfreport questionnaire that scores physical, emotional and sexual abuse and emotional and physical neglect. We calculated FA in tracts connecting regions of interest by means of diffusion magnetic resonance and gray matter segmentation from T1 images (Supplementary Material). The study complied with the Helsinki Declaration and was approved by the local ethical committee.

Using non-parametric tests, CTQ scores were compared between groups and correlations were assessed between those scores and FA in the tracts linking superior-medial prefrontal (SMPF) and rostral lateral prefrontal (RLPF) to selected regions. We restricted these calculations to tracts showing decreased FA in our former study (Molina et al. 2017) (i.e., right RLPF-cingulate, right RLPF-caudate, left SMPF-hippocampus, left SMPF-thalamus and left SMPF-caudate) and CTQ scores significantly higher in patients. To further characterizing correlates of childhood trauma, we also calculated Spearman's coefficients between CTQ scores and cognitive performance.

Scores for early adverse experiences were significantly higher in patients for emotional $(U=84, z=2.56, p=0.006)$ and physical $(U=$ $82, z=2.73, p=0.010)$ neglect and emotional abuse $(U=93, z=$ $2.44, \mathrm{p}=0.015)$. In patients, physical neglect scores were inversely correlated with FA values in left SMPF-hippocampus tract (rho = $-0.579, \mathrm{p}=0.009$; Fig. 1 ). There were no associations between FA and CTQ scores in controls. Physical neglect was directly correlated to perseverative errors in WCST (rho $=0.492, \mathrm{p}=0.005$ ) and emotional neglect was inversely correlated to working memory (rho $=-0.529$, $\mathrm{p}=0.002$ ) and performance speed (rho $=-0.501, \mathrm{p}=0.004$ ).

Neglect can be understood as the absence of the adequate signals of adequate support from the adults who care for the children. Thus, they are highly stressful to the child and likely activate the body's stress response system. Neurosteroids contribute to myelination (Garcia-Segura and Melcangi 2006) and early stress and/or neglect hampers its regulation (Brunton 2015). Therefore, the threat posed by neglect is likely to affect myelination in early life and contribute to lower FA values in key tracts in schizophrenia. In support of this possibility, lower FA values were found in adopted children raised in institutions in tracts connecting PFC and other brain regions (Hanson et al. 2013). Moreover, children raised in orphanages showed reduced FA in frontal, temporal and parietal WM, associated to duration of time in orphanage (Govindan et al. 2010). According to our data, connectivity between PFC and hippocampus is specially affected by maltreatment in schizophrenia, which seems coherent with the reduced functional hippocampal connectivity in adolescents who had suffered maltreatment (Herringa et al. 2013).

Our results preliminarily suggest a role for early neglect in miswiring of PFC in schizophrenia patients, in particular for the fronto-limbic connectivity, to be confirmed in larger samples.

\section{Conflict of interest}

None of the authors have any conflict of interest to declare.

\section{Contributors}

V. Molina designed the study and wrote the protocol. P. del Vale and P. Marqués performed clinical assessment. A. Lubeiro, R. de Luis-García performed the image analysis. V. Molina undertook the statistical analyses. A. Alvarez-Astorga, D. Ortega, M. Jiménez performed the cognitive study. All authors contributed to the article, revised and approved the final manuscript.

\section{Acknowledgements}

This study has been supported by the "Fondo de Investigaciones Sanitarias" from Instituto de Salud Carlos III (PI15/00299) and the Gerencia Regional de Salud de Castilla y León (GRS 1134/A/15 and GRS 1263/A/16) grants to V. Molina; a predoctoral research fellowship from the Consejería de Educación - Junta de Castilla to A. Lubeiro.

\section{Appendix A. Supplementary data}

Supplementary data to this article can be found online at http://dx. doi.org/10.1016/j.schres.2017.06.005.

\section{References}

Bernstein, D.P., Stein, J.A., Newcomb, M.D., Walker, E., Pogge, D., Ahluvalia, T., Stokes, J., Handelsman, L., Medrano, M., Desmond, D., Zule, W., 2003. Development and validation of a brief screening version of the childhood trauma questionnaire. Child Abuse Negl. 27 (2), 169-190.

Brunton, P.J., 2015. Programming the brain and behaviour by early-life stress: a focus on neuroactive steroids. J. Neuroendocrinol. 27 (6), 468-480. 


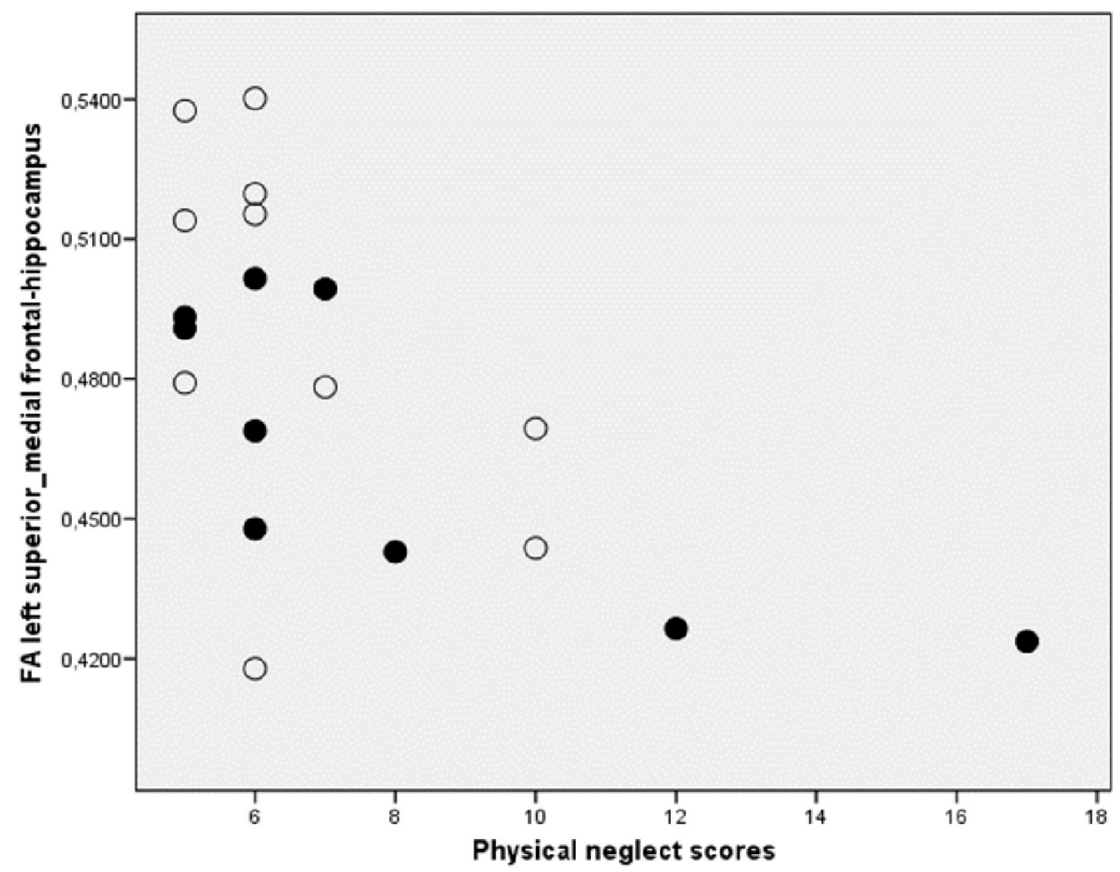

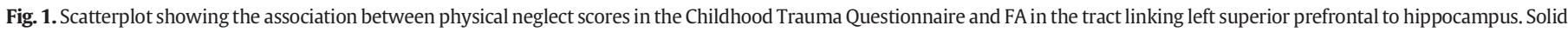
dots represent chronic patients, and open dots represent FE patients.

Davidson, R.J., McEwen, B.S., 2012. Social influences on neuroplasticity: stress and interventions to promote well-being. Nat. Neurosci. 15 (5), 689-695.

Delpech, J.C., Wei, L., Hao, J., Yu, X., Madore, C., Butovsky, O., Kaffman, A., 2016. Early life stress perturbs the maturation of microglia in the developing hippocampus. Brain Behav. Immun. 57, 79-93.

Garcia-Segura, L.M., Melcangi, R.C., 2006. Steroids and glial cell function. Glia 54 (6), 485-498.

Govindan, R.M., Behen, M.E., Helder, E., Makki, M.I., Chugani, H.T., 2010. Altered water diffusivity in cortical association tracts in children with early deprivation identified with tract-based spatial statistics (TBSS). Cereb. Cortex 20 (3), 561-569.

Hanson, J.L., Adluru, N., Chung, M.K., Alexander, A.L., Davidson, R.J., Pollak, S.D., 2013. Early neglect is associated with alterations in white matter integrity and cognitive functioning. Child Dev. 84 (5), 1566-1578.

Herringa, R.J., Birn, R.M., Ruttle, P.L., Burghy, C.A., Stodola, D.E., Davidson, R.J., Essex, M.J., 2013. Childhood maltreatment is associated with altered fear circuitry and increased internalizing symptoms by late adolescence. Proc. Natl. Acad. Sci. U. S. A. 110 (47), 19119-19124.

Molina, V., Lubeiro, A., Soto, O., Rodriguez, M., Alvarez, A., Hernandez, R., de Luis-Garcia, R. 2017. Alterations in prefrontal connectivity in schizophrenia assessed using diffusion magnetic resonance imaging. Prog. Neuro-Psychopharmacol. Biol. Psychiatry 76, 107-115.

Poletti, S., Mazza, E., Bollettini, I., Locatelli, C., Cavallaro, R., Smeraldi, E., Benedetti, F., 2015 Adverse childhood experiences influence white matter microstructure in patients with schizophrenia. Psychiatry Res. 234 (1), 35-43.

Vicente Molina

Psychiatry Department, School of Medicine, University of Valladolid, Av. Ramón y Cajal, 7, 47005 Valladolid, Spain

Psychiatry Service, Clinical Hospital of Valladolid, Ramón y Cajal, 3, 47003 Valladolid, Spain

Neurosciences Institute of Castilla y León (INCYL), Pintor Fernando Gallego, 1, 37007, University of Salamanca, Spain CIBERSAM (Biomedical Research Network in Mental Health), Instituto de Salud Carlos III, Spain Instituto de Investigación Biomédica de Salamanca (IBSAL), Spain Corresponding author at: Dept. of Psychiatry, University Hospital of Valladolid, Av. Ramón y Cajal, 7, Valladolid 48005, Spain. E-mail address: vicente.molina@uva.es
Aldara Álvarez-Astorga

Psychiatry Service, Clinical Hospital of Valladolid, Ramón y Cajal, 3, 47003

Valladolid, Spain

Alba Lubeiro

Psychiatry Department, School of Medicine, University of Valladolid, Av. Ramón y Cajal, 7, 47005 Valladolid, Spain

Daniel Ortega

Psychiatry Department, School of Medicine, University of Valladolid, Av. Ramón y Cajal, 7, 47005 Valladolid, Spain

Miguel Jiménez

Psychiatry Department, School of Medicine, University of Valladolid, Av. Ramón y Cajal, 7, 47005 Valladolid, Spain

Pilar del Valle

Psychiatry Service, Clinical Hospital of Valladolid, Ramón y Cajal, 3, 47003 Valladolid, Spain

Patricia Marqués

Psychiatry Service, Clinical Hospital of Valladolid, Ramón y Cajal, 3, 47003

Valladolid, Spain

Rodrigo de Luis-García Imaging Processing Laboratory, University of Valladolid, Paseo de Belén, 15, 47011 Valladolid, Spain

20 May 2017 